\title{
ON A PERIODIC MUTUALISM MODEL
}

\author{
YONGKUN LI
}

(Received 4 October 1996; revised 18 July 2000)

\begin{abstract}
Sufficient conditions are obtained for the existence of a globally attracting positive periodic solution of the mutualism model

$$
\begin{aligned}
& \frac{\mathrm{d} N_{1}(t)}{\mathrm{d} t}=r_{1}(t) N_{1}(t)\left[\frac{K_{1}(t)+\alpha_{1}(t) N_{2}\left(t-\tau_{2}(t)\right)}{1+N_{2}\left(t-\tau_{2}(t)\right)}-N_{1}\left(t-\sigma_{1}\right)\right], \\
& \frac{\mathrm{d} N_{2}(t)}{\mathrm{d} t}=r_{2}(t) N_{2}(t)\left[\frac{K_{2}(t)+\alpha_{2}(t) N_{1}\left(t-\tau_{1}(t)\right)}{1+N_{1}\left(t-\tau_{1}(t)\right)}-N_{2}\left(t-\sigma_{2}\right)\right],
\end{aligned}
$$

where $r_{i}, K_{i}, \alpha_{i} \in C\left(R, R^{+}\right)$and $\alpha_{i}>K_{i}, i=1,2, \tau_{i}, \sigma_{i} \in C\left(R, R_{+}\right), i=1,2$ and $r_{i}$, $K_{i}, \alpha_{i}, \tau_{i}, \sigma_{i}(i=1,2)$ are functions of period $\omega>0$.
\end{abstract}

\section{Introduction}

Consider the mutualism model

$$
\left\{\begin{array}{l}
\frac{\mathrm{d} N_{1}(t)}{\mathrm{d} t}=r_{1} N_{1}(t)\left[\frac{K_{1}+\alpha_{1} N_{2}(t)}{1+N_{2}(t)}-N_{1}(t)\right] \\
\frac{\mathrm{d} N_{2}(t)}{\mathrm{d} t}=r_{2} N_{2}(t)\left[\frac{K_{2}+\alpha_{2} N_{1}(t)}{1+N_{1}(t)}-N_{2}(t)\right]
\end{array}\right.
$$

where $r_{i}, K_{i}, \alpha_{i} \in R^{+}$are constants and $\alpha_{i}>K_{i}, i=1,2$. Depending on the nature of $K_{i}(i=1,2)$, system (1.1) can be classified as facultative, obligate or a combination of both. For more details of mutualistic interactions we refer to Vandermeer and Boucher [7], Boucher et al. [2], Dean [3], Wolin and Lawlor [8] and Boucher [1]. A

\footnotetext{
'Department of Mathematics, Yunnan University, Kunming, Yunnan 650091, People's Republic of China.

(C) Australian Mathematical Society 2001, Serial-fee code 0334-2700/01
} 
modification of system (1.1) leads to the time-lagged model

$$
\left\{\begin{array}{l}
\frac{\mathrm{d} N_{1}(t)}{\mathrm{d} t}=r_{1} N_{1}(t)\left[\frac{K_{1}+\alpha_{1} N_{2}\left(t-\tau_{2}\right)}{1+N_{2}\left(t-\tau_{2}\right)}-N_{1}(t)\right], \\
\frac{\mathrm{d} N_{2}(t)}{\mathrm{d} t}=r_{2} N_{2}(t)\left[\frac{K_{2}+\alpha_{2} N_{1}\left(t-\tau_{1}\right)}{1+N_{1}\left(t-\tau_{1}\right)}-N_{2}(t)\right],
\end{array}\right.
$$

where $\tau_{1}, \tau_{2} \in[0, \infty)$ are constants. In system (1.2) the mutualistic or cooperative effects are not realized instantaneously but take place with time delays. For further ecological applications of system (1.2), we refer to [5] and the references cited therein.

The effects of a periodically varying environment are important for evolutionary theory as the selective forces on systems in a fluctuating environment differ from those in a stable environment. Thus, the assumptions of periodicity of the parameters are a way of incorporating the periodicity of the environment (such as seasonal effects of weather, food supplies, mating habits and so forth). We refer to Pianka [6] for a discussion of the relevance of periodic environments to evolutionary theory. The purpose of this article is to consider the model

$$
\left\{\begin{array}{l}
\frac{\mathrm{d} N_{1}(t)}{\mathrm{d} t}=r_{1}(t) N_{1}(t)\left[\frac{K_{1}(t)+\alpha_{1}(t) N_{2}\left(t-\tau_{2}(t)\right)}{1+N_{2}\left(t-\tau_{2}(t)\right)}-N_{1}\left(t-\sigma_{1}(t)\right)\right], \\
\frac{\mathrm{d} N_{2}(t)}{\mathrm{d} t}=r_{2}(t) N_{2}(t)\left[\frac{K_{2}(t)+\alpha_{2}(t) N_{1}\left(t-\tau_{1}(t)\right)}{1+N_{1}\left(t-\tau_{1}(t)\right)}-N_{2}\left(t-\sigma_{2}(t)\right)\right],
\end{array}\right.
$$

together with the initial conditions:

$$
\begin{gathered}
N_{i}(t)=\varphi_{i}(t) \geq 0, \quad t \in\left[-\tau^{*}, 0\right], \quad \varphi_{i}(0)>0 \\
\varphi_{i} \in C\left(\left[-\tau^{*}, 0\right), R_{+}\right), \quad i=1,2,
\end{gathered}
$$

where $r_{i}, K_{i}, \alpha_{i} \in C\left(R, R^{+}\right), \alpha_{i}>K_{i}, i=1,2, \tau_{i}, \sigma_{i} \in C\left(R, R_{+}\right), i=1,2, r_{i}, K_{i}$, $\alpha_{i}, \tau_{i}, \sigma_{i}(i=1,2)$ are functions of period $\omega>0$ and

$$
\tau^{*}=\max _{i \leq i \leq 2}\left\{\max _{t \in[0, \omega]} \tau_{i}(t), \max _{t \in[0, \omega]} \sigma_{i}(t)\right\} .
$$

In Section 2 we discuss the existence of a positive $\omega$-periodic solution of (1.3)(1.4), in Section 3 we study the uniqueness and global attractivity of the positive periodic solution of (1.3)-(1.4) and in Section 4 we give an example to illustrate that the conditions of our results can be realized.

\section{Existence of a positive periodic solution}

In this section we use Mawhin's continuation theorem to show the existence of at least one positive periodic solution of (1.3)-(1.4). To do so, we need to introduce the following notation. 
Let $X, Y$ be real Banach spaces, $L:$ Dom $L \subset X \rightarrow Y$ a Fredholm mapping of index zero and $P: X \rightarrow X, Q: Y \rightarrow Y$ continuous projectors such that $\operatorname{Im} P=$ $\operatorname{Ker} L, \operatorname{Ker} Q=\operatorname{Im} L$ and $X=\operatorname{Ker} L \bigoplus \operatorname{Ker} P, Y=\operatorname{Im} L \bigoplus \operatorname{Im} Q$. Let $L_{P}$ denote the restriction of $L$ to $\operatorname{Dom} L \bigcap \operatorname{Ker} P, K_{P}: \operatorname{Im} L \rightarrow \operatorname{Ker} P \bigcap \operatorname{Dom} L$ the inverse (to $\left.L_{P}\right)$ and $J: \operatorname{Im} Q \rightarrow \operatorname{Ker} L$ an isomorphism of $\operatorname{Im} Q$ onto $\operatorname{Ker} L$.

For convenience, we introduce Mawhin's continuation theorem [4, page 40] as follows.

LEMMA 2.1. Let $\Omega \subset X$ be an open bounded set and $N: X \rightarrow Y$ be a continuous operator which is L-compact on $\bar{\Omega}$ (that is, $Q N: \bar{\Omega} \rightarrow Y$ and $K_{P}(l-Q) N: \bar{\Omega} \rightarrow Y$ are compact). Assume

(a) for each $\lambda \in(0,1), x \in \partial \Omega \bigcap \operatorname{Dom} L, L x \neq \lambda N x$;

(b) for each $x \in \partial \Omega \bigcap \operatorname{Ker} L, Q N x \neq 0$, and $\operatorname{deg}\{J Q N, \Omega \bigcap \operatorname{Ker} L, 0\} \neq 0$.

Then $L x=N x$ has at least one solution in $\bar{\Omega} \bigcap \operatorname{Dom} L$.

LEMMA 2.2. Let

$$
f(x, y)=\left(a_{1}-\frac{a_{1}-b_{1}}{1+e^{y}}-c_{1} e^{x}, a_{2}-\frac{a_{2}-b_{2}}{1+e^{x}}-c_{2} e^{y}\right)
$$

iand $\Omega=\left\{(x, y)^{T} \in R^{2}:|x|+|y|<M\right\}$, where $M, a_{i}, b_{i}, c_{i} \in R^{+}$are constants, c $a_{i}>b_{i}, i=1,2$, and $M>\max \left\{\left|\ln \left(a_{i} / c_{i}\right)\right|,\left|\ln \left(b_{i} / c_{i}\right)\right|, i=1,2\right\}$. Then

$$
\operatorname{deg}\{f, \Omega,(0,0)\} \neq 0 \text {. }
$$

Proof. Set

$$
H(x, y, \mu)=\left(a_{1}-\frac{a_{1}-b_{1}}{1+\mu e^{y}}-c_{1} e^{x}, a_{2}-\frac{a_{2}-b_{2}}{1+\mu e^{x}}-c_{2} e^{y}\right), \quad 0 \leq \mu \leq 1 .
$$

It is then easy to see that, for $(x, y, \mu)^{T} \in R^{2} \times[0,1]$,

and

$$
\begin{array}{lc}
a_{1}-\frac{a_{1}-b_{1}}{1+\mu e^{y}}-c_{1} e^{x} \leq a_{1}-c_{1} e^{x}<0 \quad \text { as } \quad x \geq \frac{M}{2} \\
a_{2}-\frac{a_{2}-b_{2}}{1+\mu e^{x}}-c_{1} e^{y} \leq a_{2}-c_{2} e^{y}<0 \quad \text { as } \quad y \geq \frac{M}{2} \\
a_{1}-\frac{a_{1}-b_{1}}{1+\mu e^{y}}-c_{1} e^{x} \geq b_{1}-c_{1} e^{x}>0 \quad \text { as } \quad x \leq-\frac{M}{2}
\end{array}
$$

$$
a_{2}-\frac{a_{2}-b_{2}}{1+\mu e^{x}}-c_{2} e^{y} \geq b_{2}-c_{2} e^{y}>0 \text { as } \quad y \leq-\frac{M}{2} \text {. }
$$


Hence

$$
H(x, y, \mu) \neq 0 \quad \text { for } \quad(x, y, \mu) \in \partial \Omega \times[0,1] .
$$

It follows from the property of invariance under a homotopy that

$$
\operatorname{deg}\{f(x, y), \Omega,(0,0)\}=\operatorname{deg}\{H(x, y, 0), \Omega,(0,0)\} .
$$

By a straightforward computation, we find

$$
\operatorname{deg}\{H(x, y, 0), \Omega,(0,0)\}=-1 \neq 0 .
$$

The proof is complete.

We now come to the fundamental theorem of this paper.

THEOREM 2.3. The initial value problem (1.3)-(1.4) has at least one positive $\omega$ periodic solution.

PROOF. Since solutions of (1.3)-(1.4) remain positive for $t \geq 0$, we can let

$$
x(t)=\log \left[N_{1}(t)\right] \quad \text { and } \quad y(t)=\log \left[N_{2}(t)\right]
$$

and derive that

$$
\left\{\begin{array}{l}
\frac{\mathrm{d} x(t)}{\mathrm{d} t}=r_{1}(t)\left[\frac{K_{1}(t)+\alpha_{1}(t) e^{y\left(t-\tau_{2}(t)\right)}}{1+e^{y\left(t-\tau_{2}(t)\right)}}-e^{x\left(t-\sigma_{1}(t)\right)}\right] \\
\frac{\mathrm{d} y(t)}{\mathrm{d} t}=r_{2}(t)\left[\frac{K_{2}(t)+\alpha_{2}(t) e^{x\left(t-\tau_{1}(t)\right)}}{1+e^{x\left(t-\tau_{1}(t)\right)}}-e^{y\left(t-\sigma_{2}(t)\right)}\right] .
\end{array}\right.
$$

Take

$$
X=Y=\left\{(x(t), y(t))^{T}: x(t), y(t) \in C(R, R), x(t+\omega)=x(t), y(t+\omega)=y(t)\right\}
$$
and

$$
\left\|(x, y)^{T}\right\|=\max _{0 \leq t \leq \omega}|x(t)|+\max _{0 \leq t \leq \omega}|y(t)| .
$$

With this norm, $X$ is a Banach space. Let

$$
\begin{gathered}
N\left[\begin{array}{l}
x \\
y
\end{array}\right]=\left[\begin{array}{l}
r_{1}(t)\left[\frac{K_{1}(t)+\alpha_{1}(t) e^{y\left(t-\tau_{2}(t)\right)}}{1+e^{y\left(t-\tau_{2}(t)\right)}}-e^{x\left(t-\sigma_{1}(t)\right)}\right] \\
r_{2}(t)\left[\frac{K_{2}(t)+\alpha_{2}(t) e^{x\left(t-\tau_{1}(t)\right)}}{1+e^{x\left(t-\tau_{1}(t)\right)}}-e^{y\left(t-\sigma_{2}(t)\right)}\right]
\end{array}\right], \\
L\left[\begin{array}{l}
x \\
y
\end{array}\right]=\left[\begin{array}{l}
\frac{\mathrm{d} x(t)}{\mathrm{d} t} \\
\frac{\mathrm{d} y(t)}{\mathrm{d} t}
\end{array}\right], \quad P\left[\begin{array}{l}
x \\
y
\end{array}\right]=Q\left[\begin{array}{l}
x \\
y
\end{array}\right]=\left[\begin{array}{l}
\frac{1}{\omega} \int_{0}^{\omega} x(t) \mathrm{d} t \\
\frac{1}{\omega} \int_{0}^{\omega} y(t) \mathrm{d} t
\end{array}\right], \quad\left[\begin{array}{l}
x \\
y
\end{array}\right] \in X .
\end{gathered}
$$


Since $\operatorname{Ker} L=R^{2}$ and $\operatorname{Im} L$ is closed in $X, L$ is a Fredholm mapping of index zero. Furthermore, we have that $N$ is $L$-compact on $\bar{\Omega}$ (see [4]), where $\Omega$ is any open bounded set in $X$. Corresponding to the equation $L x=\lambda N x$, we have

$$
\left\{\begin{array}{l}
\frac{\mathrm{d} x(t)}{\mathrm{d} t}=\lambda r_{1}(t)\left[\frac{K_{1}(t)+\alpha_{1}(t) e^{\left.y\left(t-\tau_{2}(t)\right)\right)}}{1+e^{y\left(t-\tau_{2}(t)\right)}}-e^{x\left(t-\sigma_{1}(t)\right)}\right], \\
\frac{\mathrm{d} y(t)}{\mathrm{d} t}=\lambda r_{2}(t)\left[\frac{K_{2}(t)+\alpha_{2}(t) e^{\left.x\left(t-\tau_{1}(t)\right)\right)}}{1+e^{x\left(t-\tau_{1}(t)\right)}}-e^{y\left(t-\sigma_{2}(t)\right)}\right] .
\end{array}\right.
$$

Assume that $(x(t), y(t))^{T} \in X$ is a solution of system (2.4) for a certain $\lambda \in(0,1)$. By integrating (2.3) over $[0, \omega]$, we obtain

$$
\int_{0}^{\omega} r_{1}(t)\left[\frac{K_{1}(t)+\alpha_{1}(t) e^{y\left(t-\tau_{2}(t)\right)}}{1+e^{y\left(t-\tau_{2}(t)\right)}}-e^{x\left(t-\sigma_{1}\right)}\right] \mathrm{d} t=0
$$

and

$$
\int_{0}^{\omega} r_{2}(t)\left[\frac{K_{2}(t)+\alpha_{2}(t) e^{x\left(t-\tau_{1}(t)\right)}}{1+e^{x\left(t-\tau_{1}(t)\right)}}-e^{y\left(t-\sigma_{2}\right)}\right] \mathrm{d} t=0 .
$$

It is easy to see that we can rewrite (2.4) and (2.5) respectively as

and

$$
\int_{0}^{\omega} \frac{r_{1}(t)\left(\alpha_{1}(t)-K_{1}(t)\right)}{1+e^{y\left(t-\tau_{2}(t)\right)}} \mathrm{d} t+\int_{0}^{\omega} r_{1}(t) e^{x\left(t-\sigma_{1}\right)} \mathrm{d} t=\int_{0}^{\omega} r_{1}(t) \alpha_{1}(t) \mathrm{d} t
$$

$$
\int_{0}^{\omega} \frac{r_{2}(t)\left(\alpha_{2}(t)-K_{2}(t)\right)}{1+e^{x\left(t-\tau_{1}(t)\right)}} \mathrm{d} t+\int_{0}^{\omega} r_{2}(t) e^{y\left(t-\sigma_{2}\right)} \mathrm{d} t=\int_{0}^{\omega} r_{2}(t) \alpha_{2}(t) \mathrm{d} t .
$$

Thus from (2.3) and (2.6), it follows that

$$
\begin{aligned}
\int_{0}^{\omega}\left|x^{\prime}(t)\right| \mathrm{d} t & <\lambda \int_{0}^{\omega} r_{1}(t)\left[\frac{K_{1}(t)+\alpha_{1}(t) e^{y\left(t-\tau_{2}(t)\right)}}{1+e^{y\left(t-\tau_{2}(t)\right)}}+e^{x\left(t-\sigma_{1}\right)}\right] \mathrm{d} t \\
& <\int_{0}^{\omega} r_{1}(t) \alpha_{1}(t) \mathrm{d} t+\int_{0}^{\omega} \frac{r_{1}(t)\left(\alpha_{1}(t)-K_{1}(t)\right)}{1+e^{y\left(t-\tau_{2}(t)\right)}} \mathrm{d} t+\int_{0}^{\omega} r_{1}(t) e^{x\left(t-\sigma_{1}\right)} \mathrm{d} t \\
& =2 \int_{0}^{\omega} r_{1}(t) \alpha_{1}(t) \mathrm{d} t \stackrel{\text { def }}{=} M_{1},
\end{aligned}
$$

that is,

$$
\int_{0}^{\omega}\left|x^{\prime}(t)\right| \mathrm{d} t<M_{1}
$$

Similarly, by (2.3) and (2.7) we have

$$
\int_{0}^{\omega}\left|y^{\prime}(t)\right| \mathrm{d} t<2 \int_{0}^{\omega} r_{2}(t) \alpha(t) \mathrm{d} t \stackrel{\text { def }}{=} M_{2} .
$$

Moreover, from (2.6) it follows that

$$
\int_{0}^{\omega} r_{1}(t) \alpha_{1}(t) \mathrm{d} t \geq \int_{0}^{\omega} r_{1}(t) e^{x\left(t-\sigma_{1}\right)} \mathrm{d} t \geq \int_{0}^{\omega} r_{1}(t) K_{1}(t) \mathrm{d} t
$$


which implies that there exists a point $t_{1}^{\prime} \in[0, \omega]$ and a constant $C_{1}>0$ such that

$$
\left|x\left(t_{1}^{\prime}-\sigma_{1}\left(t_{1}^{\prime}\right)\right)\right|<C_{1} .
$$

Suppose that $t_{1}^{\prime}-\sigma_{1}\left(t_{1}^{\prime}\right)=t_{1}+n \omega, t_{1} \in[0, \omega]$ and $n$ is an integer, then

$$
\left|x\left(t_{1}\right)\right|<C_{1} .
$$

Similarly, by (2.7) we can obtain that there exists a point $t_{2} \in[0, \omega]$ and a constant $C_{2}>0$ such that

$$
\left|y\left(t_{2}\right)\right|<C_{2}
$$

Therefore it follows from (2.8)-(2.11) that

$$
\begin{aligned}
& \max _{r \in[0, \omega]}|x(t)| \leq\left|x\left(t_{1}\right)\right|+\int_{0}^{\omega}\left|x^{\prime}(t)\right| \mathrm{d} t<C_{1}+M_{1}, \\
& \max _{t \in[0, \omega]}|y(t)| \leq\left|y\left(t_{1}\right)\right|+\int_{0}^{\omega}\left|y^{\prime}(t)\right| \mathrm{d} t<C_{2}+M_{2} .
\end{aligned}
$$

Clearly $M_{i}$ and $C_{i}(i=1,2)$ are independent of $\lambda$. Denote $M=M_{1}+M_{2}+C_{1}+C_{2}+D$, where $D>0$ is taken sufficiently large such that $M>\max \left\{\left|\ln \left(a_{i} / c_{i}\right)\right|,\left|\ln \left(b_{i} / c_{i}\right)\right|\right.$, $i=1,2\}$. Now we take $\Omega=\left\{(x(t), y(t))^{T} \in X:\left\|(x, y)^{T}\right\|<M\right\}$. This satisfies condition (a) in Lemma 2.1.

When $(x, y)^{T} \in \partial \Omega \bigcap \operatorname{Ker} L=\partial \Omega \bigcap R^{2},(x, y)^{T}$ is a constant vector in $R^{2}$ with $|x|+|y|=M$. Then

where

$$
Q N\left[\begin{array}{l}
x \\
y
\end{array}\right]=\left[\begin{array}{l}
\overline{r_{1} \alpha_{1}}-\frac{\overline{r_{1} \alpha_{1}}-\overline{r_{1} K_{1}}}{1+e^{y}}-\bar{r}_{1} e^{x} \\
\overline{r_{2} \alpha_{2}}-\frac{\overline{r_{2} \alpha_{2}}-\overline{r_{2} K_{2}}}{1+e^{x}}-\bar{r}_{2} e^{y}
\end{array}\right] \neq\left[\begin{array}{l}
0 \\
0
\end{array}\right],
$$

$$
\bar{r}_{i}=\frac{1}{\omega} \int_{0}^{\omega} r_{i}(t) \mathrm{d} t, \quad \overline{r_{i} \alpha_{i}}=\frac{1}{\omega} \int_{0}^{\omega} r_{i}(t) \alpha_{i}(t) \mathrm{d} t, \quad \overline{r_{i} K_{i}}=\frac{1}{\omega} \int_{0}^{\omega} r_{i}(t) K_{i}(t) \mathrm{d} t,
$$

$i=1$, 2. Furthermore, take $J=I: \operatorname{Im} Q \rightarrow \operatorname{Ker} L,(x, y)^{T} \mapsto(x, y)^{T}$. By Lemma 2.2, we have

$$
\operatorname{deg}\left\{J Q N(x, y)^{T}, \Omega,(0,0)\right\}=\operatorname{deg}\left\{Q N(x, y)^{T}, \Omega,(0,0)\right\} \neq 0 .
$$

We now know that $\Omega$ verifies all the requirements in Lemma 2.1 and thus that (2.2) has at least one $\omega$-periodic solution. By (2.1), we easily see that (1.3) -(1.4) has at least one positive $\omega$-periodic solution. The proof is complete. 


\section{Uniqueness and global attractivity}

We first obtain certain upper and lower estimates for solutions of (1.3)-(1.4). For convenience we introduce the notation:

$$
\begin{aligned}
r_{11} & =\max _{t \in[0, \omega]} r_{1}(t), & r_{21} & =\max _{t \in[0, \omega]} r_{2}(t), \\
\alpha_{11} & =\max _{t \in[0, \omega]} \alpha_{1}(t), & \alpha_{21} & =\max _{t \in[0, \omega]} \alpha_{2}(t), \\
K_{12} & =\min _{t \in[0, \omega]} K_{1}(t), & K_{22} & =\min _{t \in[0, \omega]} K_{2}(t), \\
\sigma_{11} & =\max _{t \in[0, \omega]} \sigma_{1}(t), & \sigma_{21} & =\max _{t \in[0, \omega]} \sigma_{2}(t) .
\end{aligned}
$$

LEMMA 3.1. If $\left(N_{1}(t), N_{2}(t)\right)$ is a solution of the initial value problem (1.3)-(1.4) then there exist numbers $T_{1}$ and $T_{2}$ such that

$$
B_{1} \leq N_{1}(t) \leq A_{1} \quad \text { for } t \geq T_{1}
$$

and

$$
B_{2} \leq N_{2}(t) \leq A_{2} \text { for } t \geq T_{2} \text {, }
$$

in which $A_{1}=\alpha_{11} \exp \left(\alpha_{11} r_{11} \sigma_{11}\right), A_{2}=\alpha_{21} \exp \left(\alpha_{21} r_{21} \sigma_{21}\right), B_{1}=K_{12} \exp \left[r_{11} \sigma_{11}\left(K_{12}-\right.\right.$ $\left.\left.A_{1}\right)\right]$ and $B_{2}=K_{22} \exp \left[r_{21} \sigma_{21}\left(K_{22}-A_{1}\right)\right]$.

PROOF. It is easy to see that $N_{1}$ and $N_{2}$ satisfy

$$
\left\{\begin{array}{l}
\frac{\mathrm{d} N_{1}(t)}{\mathrm{d} t} \leq r_{1}(t) N_{1}(t)\left[\alpha_{11}-N_{1}\left(t-\sigma_{1}(t)\right)\right] \\
\frac{\mathrm{d} N_{2}(t)}{\mathrm{d} t} \leq r_{2}(t) N_{2}(t)\left[\alpha_{21}-N_{2}\left(t-\sigma_{2}(t)\right)\right]
\end{array}\right.
$$

Now either $N_{1}(t)$ is oscillatory about $\alpha_{11}$ or it is nonoscillatory. In the case where $N_{1}(t)$ is oscillatory about $\alpha_{11}$, we let $\left\{t_{n}\right\}$ be the sequence such that $\lim _{n \rightarrow \infty} t_{n}=\infty$ and $\alpha_{11}-N_{1}\left(t_{n}\right)=0$. Let $N_{1}\left(t_{n}^{*}\right)$ be the local maximum of $N_{1}(t)$ on $\left(t_{n}, t_{n+1}\right)$. Then

$$
0=N_{1}^{\prime}\left(t_{n}^{*}\right) \leq r_{1}\left(t_{n}^{*}\right) N_{1}\left(t_{n}^{*}\right)\left[\alpha_{11}-N_{1}\left(t_{n}^{*}-\sigma_{1}\left(t_{n}^{*}\right)\right)\right]
$$

Now $N_{1}\left(t_{n}^{*}-\sigma_{1}\left(t_{n}^{*}\right)\right) \leq \alpha_{11}$; so let $\xi$ be the zero of $\alpha_{11}-N_{1}(t)$ in $\left[t_{n}^{*}-\sigma_{1}\left(t_{n}^{*}\right), t_{n}^{*}\right]$. By integrating (3.1) from $\xi$ to $t_{n}^{*}$, we have

$$
\log \frac{N_{1}\left(t_{n}^{*}\right)}{N_{1}(\xi)} \leq \int_{\xi}^{t_{n}^{*}} \alpha_{11} r_{1}(t) \mathrm{d} t,
$$


or

$$
N_{1}\left(t_{n}^{*}\right) \leq \alpha_{11} \exp \left[\alpha_{11} \int_{\xi}^{t_{n}^{*}} r_{1}(t) \mathrm{d} t\right] \leq \alpha_{11} \exp \left(\alpha_{11} r_{11} \sigma_{11}\right)
$$

that is,

$$
N_{1}(t) \leq \alpha_{11} \exp \left(\alpha_{11} r_{11} \sigma_{11}\right)=A_{1} \quad \text { for } \quad \mathrm{t} \geq \mathrm{t}_{1}+2 \sigma_{11}
$$

Next suppose that $N_{1}(t)$ is nonoscillatory about $\alpha_{11}$. Then it is easy to see that for every $\varepsilon>0$ there exists a $T_{1}^{\prime}=T_{1}^{\prime}(\varepsilon)$ such that

$$
N_{1}(t)<\alpha_{11}+\varepsilon \quad \text { for } t>T_{1}^{\prime}
$$

This together with (3.2) implies that there exists a $T_{2}^{\prime}$ such that

$$
N_{1}(t) \leq A_{1} \text { for } t>T_{2}^{\prime}
$$

On the other hand, from (1.3) we find

$$
\left\{\begin{array}{l}
\frac{\mathrm{d} N_{1}(t)}{\mathrm{d} t} \geq r_{1}(t) N_{1}(t)\left[K_{12}-N_{1}\left(t-\sigma_{1}(t)\right)\right] \\
\frac{\mathrm{d} N_{2}(t)}{\mathrm{d} t} \geq r_{2}(t) N_{2}(t)\left[K_{22}-N_{2}\left(t-\sigma_{2}(t)\right)\right]
\end{array}\right.
$$

Let $N_{1}(t)$ be an oscillatory solution about $K_{12}$ and let $\left\{s_{n}\right\}$ be a sequence such that $\lim _{n \rightarrow \infty} s_{n}=\infty$ and $\left.N_{(} s_{n}\right)-K_{12}=0$. Suppose that $N_{1}\left(s_{n}^{*}\right)$ is a local minimum of $N_{1}(t)$ on $\left(s_{n}, s_{n+1}\right)$. Then

$$
0=N_{1}^{\prime}\left(s_{n}^{*}\right) \geq r_{1}\left(s_{n}^{*}\right) N_{1}\left(s_{n}^{*}\right)\left[K_{12}-N_{1}\left(s_{n}^{*}-\sigma_{1}\left(s_{n}^{*}\right)\right)\right] .
$$

So $K_{12}-N_{1}\left(s_{n}^{*}-\sigma_{1}\left(s_{n}^{*}\right)\right) \leq 0$, that is, there exists a point $\eta \in\left[s_{n}^{*}-\sigma_{1}\left(s_{n}^{*}\right), s_{n}^{*}\right]$ such that $N_{1}(\eta)=K_{12}$. Note that $K_{12}-A_{1}<0$, then

$$
\log \frac{N_{1}\left(s_{n}^{*}\right)}{K_{12}} \geq \int_{\eta}^{s_{n}^{*}} r_{1}(t)\left(K_{12}-A_{1}\right) \mathrm{d} t \geq r_{11} \sigma_{11}\left(K_{12}-A_{1}\right)
$$

Hence

$$
N_{1}\left(s_{n}^{*}\right) \geq K_{12} \exp \left[r_{11} \sigma_{11}\left(K_{12}-A_{1}\right)\right]
$$

that is,

$$
N_{1}(t) \geq K_{12} \exp \left[r_{11} \sigma_{11}\left(K_{12}-A_{1}\right)\right]=B_{1} \quad \text { for } t \geq t_{1}+2 \sigma_{11}
$$

Next, suppose that $N_{1}(t)$ is nonoscillatory about $K_{12}$. One can easily prove in this case that for every positive $\varepsilon$ there exists a $T_{3}^{\prime}=T_{3}^{\prime}(\varepsilon)$ such that

$$
N_{1}(t)>K_{12}-\varepsilon \quad \text { for } t \geq T_{3}^{\prime} \text {. }
$$


This together with (3.4) implies that there exists a $T_{4}^{\prime}$ such that

$$
N_{1}(t) \geq B_{1} \quad \text { for } \quad \mathrm{t} \geq \mathrm{T}_{4}
$$

Similarly, one can prove that there exists a $T_{2}$ such that

$$
B_{2} \leq N_{2}(t) \leq A_{2} \text { for } t \geq T_{2}
$$

The proof of Lemma 3.1 is complete.

We will now proceed to derive sufficient conditions under which (1.3)-(1.4) has a unique positive $\omega$-periodic solution $\left(N_{1}^{*}(t), N_{2}^{*}(t)\right)$ which globally attracts all other positive solutions of (1.3)-(1.4).

Let $\left(N_{1}^{*}(t), N_{2}^{*}(t)\right)$ be a positive $\omega$-periodic solution of (1.3)-(1.4), whose existence is given by Theorem 2.1 . We set

$$
N_{1}(t)=N_{1}^{*}(t) e^{x(t)} \quad \text { and } \quad N_{2}(t)=N_{2}^{*}(t) e^{y(t)}
$$

and derive that

where

$$
\left\{\begin{array}{l}
\frac{\mathrm{d} x(t)}{\mathrm{d} t}=F\left[x\left(t-\sigma_{1}(t)\right), y\left(t-\tau_{2}(t)\right)\right]-F(0,0), \\
\frac{\mathrm{d} y(t)}{\mathrm{d} t}=G\left[x\left(t-\tau_{1}(t)\right), y\left(t-\sigma_{2}(t)\right)\right]-G(0,0),
\end{array}\right.
$$

$$
\begin{aligned}
& F(u, v)=-N_{1}^{*}\left(t-\sigma_{1}(t)\right) e^{u}-\frac{\alpha_{1}(t)-K_{1}(t)}{1+N_{2}^{*}\left(t-\tau_{2}(t)\right) e^{v}} \\
& G(u, v)=-N_{2}^{*}\left(t-\sigma_{2}(t)\right) e^{v}-\frac{\alpha_{2}(t)-K_{2}(t)}{1+N_{1}^{*}\left(t-\tau_{1}(t)\right) e^{u}}
\end{aligned}
$$

By the mean value theorem of differential calculus, we can rewrite (3.6) in the form

where

$$
\left\{\begin{array}{l}
\frac{\mathrm{d} x(t)}{\mathrm{d} t}=-a_{11}(t) x\left(t-\sigma_{1}(t)\right)+a_{12}(t) y\left(t-\tau_{2}(t)\right), \\
\frac{\mathrm{d} y(t)}{\mathrm{d} t}=a_{21}(t) x\left(t-\tau_{1}(t)\right)-a_{22}(t) y\left(t-\sigma_{2}(t)\right),
\end{array}\right.
$$

$$
\begin{array}{ll}
a_{11}(t)=\eta_{1}(t), & a_{12}(t)=\frac{\left(\alpha_{1}(t)-K_{1}(t)\right) \eta_{2}(t)}{\left(1+\eta_{2}(t)\right)^{2}} \\
a_{22}(t)=\eta_{3}(t), & a_{21}(t)=\frac{\left(\alpha_{2}(t)-K_{2}(t)\right) \eta_{4}(t)}{\left(1+\eta_{4}(t)\right)^{2}}
\end{array}
$$

and $\eta_{1}(t)$ lies between $N_{1}^{*}\left(t-\sigma_{1}(t)\right)$ and $N_{1}\left(t-\sigma_{1}(t)\right), \eta_{2}(t)$ lies between $N_{2}^{*}\left(t-\tau_{2}(t)\right)$ and $N_{2}\left(t-\tau_{2}(t)\right), \eta_{3}(t)$ lies between $N_{2}^{*}\left(t-\sigma_{2}(t)\right)$ and $N_{2}\left(t-\sigma_{2}(t)\right)$, and $\dot{\eta}_{4}(t)$ lies 
between $N_{1}^{*}\left(t-\tau_{1}(t)\right)$ and $N_{1}\left(t-\tau_{1}(t)\right)$. By Lemma 3.1, we can conclude that there exists a number $T^{*}$ such that for all $t \geq T^{*}$, we have

$$
\begin{array}{ll}
B_{1} \leq a_{11}(t) \leq A_{1}, & \frac{\left(\alpha_{1}(t)-K_{1}(t)\right) B_{2}}{\left(1+A_{2}\right)^{2}} \leq a_{12}(t) \leq \frac{\left(\alpha_{1}(t)-K_{1}(t)\right) A_{2}}{\left(1+B_{2}\right)^{2}} \leq C_{12}, \\
B_{2} \leq a_{22}(t) \leq A_{2}, & \frac{\left(\alpha_{2}(t)-K_{2}(t)\right) B_{1}}{\left(1+A_{1}\right)^{2}} \leq a_{21}(t) \leq \frac{\left(\alpha_{2}(t)-K_{2}(t)\right) A_{1}}{\left(1+B_{1}\right)^{2}} \leq C_{21}
\end{array}
$$

where

$$
C_{12}=\frac{\left(a_{11}-K_{12}\right) A_{2}}{\left(1+B_{2}\right)^{2}}, \quad C_{21}=\frac{\left(a_{21}-K_{22}\right) A_{1}}{\left(1+B_{1}\right)^{2}} .
$$

With the above preparation we formulate our second fundamental result.

THEOREM 3.2. Assume that every solution of (3.7)-(3.8) satisfies

$$
\lim _{t \rightarrow \infty}\left[x^{2}(t)+y^{2}(t)\right]=0 .
$$

Then there exists a unique positive $\omega$-periodic solution $\left(N_{1}^{*}(t), N_{2}^{*}(t)\right)$ of $(1.3)-(1.4)$ such that all other positive solutions of (1.3)-(1.4) satisfy

$$
\lim _{t \rightarrow \infty}\left\{N_{1}(t), N_{2}(t)\right\}=\left\{N_{1}^{*}(t), N_{2}^{*}(t)\right\}
$$

PROOF. The existence of at least one positive $\omega$-periodic solution of (1.3)-(1.4) is a consequence of Theorem 2.1. The uniqueness of the periodic solution will follow from (3.10). But every solution $(x(t), y(t))$ of (3.7)-(3.8) satisfies (3.9), which implies (3.10). This completes the proof.

Next, assume that $\tau_{i}(t) \equiv \tau_{i}, \sigma_{i}(t) \equiv \sigma_{i}(i=1,2)$ are constants, and define two numbers $\mu_{1}^{*}$ and $\mu_{2}^{*}$ which satisfy

$$
\begin{aligned}
\mu_{1}^{*}= & B_{1}-\left[A_{1}\left(A_{1} \sigma_{1}+C_{12} \tau_{2}\right)+C_{21}\left(C_{21} \tau_{1}+A_{2} \sigma_{2}\right)+A_{1} \sigma_{1}\left(A_{1}+C_{12}\right)\right. \\
& \left.+C_{21} \tau_{1}\left(C_{21}+A_{2}\right)\right], \\
\mu_{2}^{*}= & B_{2}-\left[C_{12}\left(A_{1} \sigma_{1}+C_{12} \tau_{2}\right)+C_{21}\left(C_{21} \tau_{1}+A_{2} \sigma_{2}\right)+C_{12} \tau_{2}\left(A_{1}+C_{12}\right)\right. \\
& \left.+A_{2} \sigma_{2}\left(C_{21}+A_{2}\right)\right]
\end{aligned}
$$

Then we have the following result.

COROLlaRY 3.3. Assume the following conditions hold:

(i) $\tau_{i}(t) \equiv \tau_{i}, \sigma_{i}(t) \equiv \sigma_{i}(i=1,2)$ are constants;

(ii) $\mu_{1}^{*}>0, \mu_{2}^{*}>0$; 
(iii) the quadratic form

$$
Q(x, y)=[x, y]\left[\begin{array}{cc}
B_{1} & -\left(C_{12}+C_{21}\right) \\
-\left(C_{12}+C_{21}\right) & B_{2}
\end{array}\right]\left[\begin{array}{l}
x \\
y
\end{array}\right]
$$

is nonnegative.

Then (1.3)-(1.4) has a unique positive $\omega$-periodic solution $\left(N_{1}^{*}(t), N_{2}^{*}(t)\right)$, and all other positive solutions of (1.3)-(1.4) satisfy (3.10).

PROOF. Entirely similar to the proof of [5, Theorem 4.3.6], one can obtain that every solution of (3.7)-(3.8) satisfies (3.9). Therefore the conclusions of the theorem follow from Theorem 3.1. The proof is complete.

\section{An example}

Finally, as an application of our main results, we consider the system

$$
\left\{\begin{aligned}
\frac{\mathrm{d} N_{1}(t)}{\mathrm{d} t}= & \left(\frac{1}{2}+\frac{1}{2} \sin ^{2}\left(t+\phi_{1}\right)\right) N_{1}(t) \\
& \times\left[\frac{100+\frac{1}{2} \cos ^{2} t+\left(101+\sin ^{2} t\right) N_{2}\left(t-e^{-100}\right)}{1+N_{2}\left(t-e^{-100}\right)}-N_{1}\left(t-e^{-100}\right)\right], \\
\frac{\mathrm{d} N_{2}(t)}{\mathrm{d} t}= & \left(\frac{1}{2}+\frac{1}{2} \cos ^{2}\left(t+\phi_{2}\right)\right) N_{2}(t) \\
& \times\left[\frac{100+\frac{1}{2} \sin ^{2} t+\left(101+\cos ^{2} t\right) N_{1}\left(t-e^{-100}\right)}{1+N_{1}\left(t-e^{-100}\right)}-N_{2}\left(t-e^{-100}\right)\right],
\end{aligned}\right.
$$

together with the initial conditions (1.4), where $\phi_{i}, i=1,2$, are constants. One can easily verify that (4.1)-(1.4) satisfies all the conditions of Corollary 3.1. Therefore, system (4.1) has a unique positive $\omega$-periodic solution, which attracts all other positive solutions of (4.1)-(1.4).

\section{Acknowledgments}

This work was partially supported by the ABF of Yunnan Province of China. The author is grateful to a referee for his careful reading of the manuscript and his suggestions for improving the presentation of its contents. 


\section{References}

[1] D. H. Boucher, The biology of mutualism: Ecology and evolution (Croom Helm, London, 1985).

[2] D. H. Boucher, S. James and K. H. Keeler, "The ecology of mutualism", Ann. Rev. Syst. 13 (1982) 315-347.

[3] A. M. Dean, "A simple model of mutualism", Amer. Natural. 121 (1983) 409-417.

[4] R. E. Gaines and J. L. Mawhin, Coincidence degree and nonlinear differential equations, Lecture Notes in Math. 568 (Springer, Berlin, 1977).

[5] K. Gopalsamy, Stability and oscillations in delay differential equations of population dynamics (Kluwer Academic Publishers, Boston, 1992).

[6] E. R. Pianka, Evolutionary ecology (Harper and Row, New York, 1974).

[7] J. H. Vandermeer and D. H. Boucher, "Varieties of mutualistic interaction models", J. Theor. Biol. 74 (1978) 549-558.

[8] C. L. Wolin and L. R. Lawlor, "Models of facultative mutualism: density effects", Amer. Natural. 144 (1984) 843-862. 